\title{
Cultural Relics Protection System Based on ZigBee and WiFi
}

\author{
Shun $\mathrm{Na}^{1}$, Qiong $\mathrm{Wu}^{1}$, Xiaowei Qiang ${ }^{1}$, Yang $\mathrm{Liu}^{1}$, XiaoQing $\mathrm{Wu}^{2, *}$ \\ 1. College of Electronic Information Engineering, Inner Mongolia University, Hohhot, China \\ 2. College of Physics and Electronic Information, Inner Mongolia Normal University, Hohhot, China \\ nashunimu@163.com, qiongwuimu@163.com, qiangxiaoweiimu@163.com,yangliuimu@163.com, ailiyawu@163.com
}

\begin{abstract}
Cultural relics are valuable society resource for the countries. In order to protect the cultural relics effectively, a cultural relics protection system based on ZigBee and WiFi is proposed in this paper. The system is based on a wireless sensor network that collects information about the surrounding environment and of the cultural relics through sensors and detects any position changes and temperature or humidity anomalies of the monitored cultural relics. When the position of the monitored cultural relics changes or the temperature and humidity are not conducive to the storage of cultural relics, the system will send an alarm to the control center to inform the staff to handle the situation accordingly. The detailed implementation of the system is demonstrated.
\end{abstract}

Keywords-ZigBee; WiFi; cultural relics protection system; wireless sensor network; CC2530

\section{INTRODUCTION}

China, as one of the four ancient civilizations in the world, has a myriad of cultural relics. The protection of cultural relics is a complicated and formidable task. As technology continues to move forward, the means of theft used by the criminals improve as well, hence, posing enormous challenges to the protection of cultural relics. The use of a wireless sensor network in the protection of cultural relic can not only resolve many of the inconveniences of manual monitoring but also lower the power consumption, and ease the installation and configuration, hence, lowering the maintenance cost. It would provide the user with great convenience and efficiency, as well as substantial saving of resources and costs.

Some of the anti-theft protection systems employed here and abroad use cameras for monitoring. The advantages of using cameras in monitoring are that it is in real-time and it provides a record, but this method is more operator dependent and the cameras are exposed outside the monitored environment which can be easily discovered and destroyed by the criminals [1]. In addition, some of the existing alarm systems use pressure sensors, switch sensors, infrared sensors, ultrasonic sensors and acoustic sensors [2]. Pressure sensors and switch sensors are simple in construction and easy to install, but are hampered by a high rate of false responses and missing responses. Infrared sensors have a poor performance, a low sensitivity, and also a high false alarm rate. Acoustic sensors perform their anti-theft function by acquiring the acoustic signals of the footsteps, they also have the disadvantages of inaccurate acquisition [3 6].
This design of the cultural relics protection system presented here is based on ZigBee and $\mathrm{WiFi}$, and the implementation methods of the hardware and software system have been investigated. The hardware solution chooses to use the ZigBee wireless communication network to collect cultural relics protection data. The ZigBee self-organizing node network has low cost and low power consumption, and it is well suited for a cultural relics protection system [7], [8]. An embedded ARM platform is selected as the gateway of the cultural relics protection system. The software solution involves software development of ZigBee coordinator and terminal, the design of ARM gateway software, and the development of host computer software based on C\#.

\section{SYSTEM COMPOSITION PRINCIPLE}

The ZigBee and WiFi-based cultural relics protection system can be divided mainly into three parts, namely ZigBee wireless communication network, ARM gateway and PC software design. The ZigBee wireless communication network consists of four ZigBee terminal devices (the four terminal nodes are equipped respectively with a smoke sensor, a temperature and humidity sensor, a human body infrared sensor, and an ultrasonic range sensor) and a coordinator to realize the function of monitoring the surrounding environment of cultural relics for gas concentration, passive infrared, temperature and humidity and distance. The ZigBee coordinator is connected to the ARM gateway through a serial port, thereby realizing the function of the ARM to transmit the data collected by the sensors to the cloud server through the WiFi module. The ARM processor determines whether the cultural relics had moved based on the distance values collected by the ultrasonic ranging sensors. If the cultural relics had moved, the GSM module will send out a short message to notify the relevant users.

The smoke sensor can detect the gas capacity value of the environment in which the cultural relics are located in real time. It can detect timely abnormal conditions such as fires to prevent cultural relics from being damaged. The node can be placed in the same position as the temperature and humidity sensor node near the cultural relics. The temperature and humidity sensor can monitor the temperature and humidity of the environment where the protected cultural relics are located in real time. By setting the threshold value, abnormal temperature and humidity can be avoided to affect the storage of cultural relics; the human infrared sensor node is placed close to the cultural relics, so that the viewer can be guaranteed. 
The ultrasonic sensor is used to measure the distance between the node and the protected cultural object. By setting a distance threshold, if the distance between the protected cultural object and the node exceeds the threshold, that is, the protected cultural object is moved. At this time, the sensor node will transmit the information of the distance change to the upper computer alarm, thereby preventing the cultural relics from being stolen. Each sensor node transmits the collected information to the upper computer through the coordinator, and displays it through the upper computer interface, which is convenient for real-time monitoring by management personnel.

\section{A. Design of ZigBee Communication Network}

ZigBee is a low-power personal area network protocol based on the IEEE802.15.4 standard, which is a commonly used wireless communication technology [9]. ZigBee features low transmission data rate, short transmission distance, low complexity, low power consumption and low cost. These features make it widely used in remote control and automatic control [10], [11].

ZigBee has three network topologies: a star-shaped mesh structure, a mesh-type structure, and a tree-shaped mesh structure. The cultural relics protection system adopts a starshaped mesh structure. Star network is the simplest structure. In the cultural relics protection system, the amount of data needed to be collected is not large. The ZigBee network structure is built indoor. The distance of each ZigBee node is about 10 meters. The star network topology structure can meet the requirements of the system.

The coordinator is connected to the ARM gateway through the serial port, and the data collected by the four sensors can be transmitted to the gateway. CC2530 is chosen as the main board of the coordinator, because CC2530 takes 8051 chip as the main body. Developers just need to be familiar with the pin functions of the single chip microcontroller to program and shorten the development cycle of the system. Coordinator node is responsible for mobilizing the work of each sensor node, so its stability is particularly important for the whole network. Because of the large amount of data stored, CC2530 chip with large memory is chosen as the processor of the coordinator. After the four terminal nodes and one ZigBee coordinator are powered up, the coordinator node broadcasts a request command. After receiving the command, the terminal node collects data by determining the command, and the terminal node sends the data collected by the sensors to the coordinator device on-demand.

Generally speaking, a network will be created after the coordinator device is powered on and waits for the ZigBee terminal to join the network. It completes the construction of the ZigBee network topology structure after the ZigBee terminal device joins the network.

\section{B. Design of Gateway of Cultural Relics Protection System}

The ARM gateway is the communication hub of the entire cultural relics protection system, and plays an important role in the connection between the ZigBee communication network and the host computer. The gateway consists of an STM32 processor, a Wi-Fi module and a GSM module. The ARM gateway adopts a STM32F103VET6 processor to achieve the networking function of the ARM gateway through the $\mathrm{WiFi}$ module. The above-mentioned coordinator, STM32 and cloud server terminal employ a wireless communication mode, and the data is transmitted to the cloud server terminal through the ESP8266 module (Wi-Fi module). When the distance from the ultrasonic sensor to the cultural relic is abnormal, the gateway sends a short message to the persons in charge to take appropriate action. The cloud server directly uses the "lewei IOT" server which is a high-concurrency access server for solving the cloud storage. It can simultaneously complete massive sensor data access and storage tasks, ensuring that data can be safely stored in the Internet. Advanced authentication systems and security mechanisms ensure that data is only shared within the allowed range. The system analyzes and processes the received data on the cloud server, thereby realizing the function of remotely viewing the collected data through the host computer in anywhere the network can be connected. At the same time, since the WiFi module sends data to the cloud server based on the HTTP protocol, an HTTP connection request needs to be initiated on the host computer client. The ARM gateway connects to the ZigBee coordinator through the serial port, finally monitoring the ZigBee network by the host computer. Fig. 1 below shows the structure of the ARM gateway of the cultural relics protection system.

\section{Design of Host Computer Program}

The cultural relics protection system achieves the function of real-time remote monitoring of the surrounding environment of cultural relics by writing corresponding host computer programs. The host computer directly uses PC to avoid unnecessary steps to develop DSP. By using the graphical programming software LABVIEW, the developer develops the corresponding host computer software to analyze and process the received data. The system uses the $\mathrm{C \#}$ language to develop the host computer client, and the host computer is connected to the cloud server through the HTTP protocol, thereby achieving the real-time remote monitoring function of the cultural relics protection system.

\section{SyStem HARDWARE DESIGN AND REALIZATION}

The cultural relics protection system has three major components, ZigBee wireless communication network, cultural relic protection system ARM gateway and host computer. The system framework is shown in Fig. 2. In this system, the ZigBee communication network is composed of four ZigBee terminal devices and one coordinator. All five ZigBee modules used in the ZigBee communication network are CC2530 chips. Each terminal node carries different sensor modules to collect data, and the coordinator is connected to the ARM gateway through a serial port. The ARM gateway uses a STM32F103VET6 as the processor, and transmits the data to the cloud server through a serial port. The data are transmitted to the cloud server through a WiFi module and under HTTP protocol. The host computer accesses the cloud server through the HTTP protocol, thus achieving the function of remotely monitoring the cultural relics. 


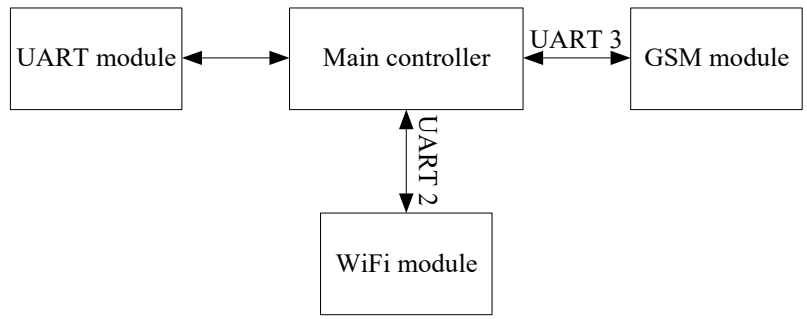

Fig. 1. ARM gateway structure of cultural relics protection system.

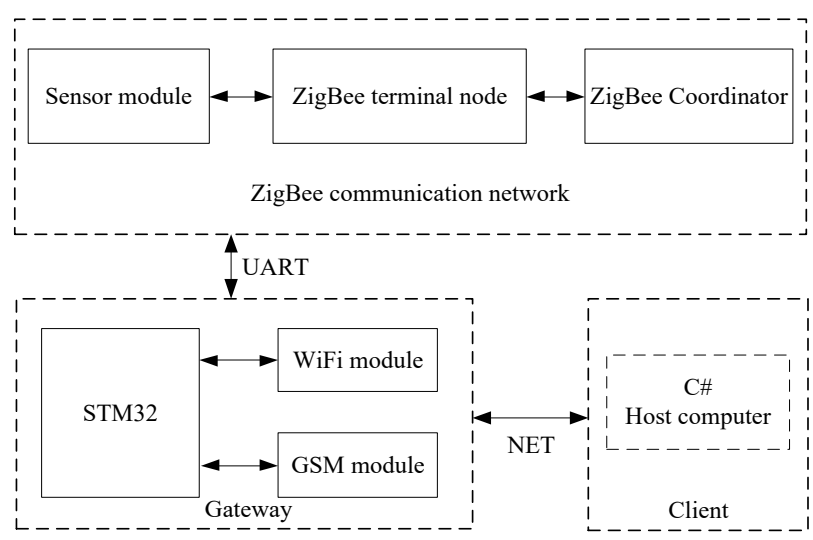

Fig. 2. Block diagram of cultural relics protection system.

\section{A. Hardware design of ZigBee module}

CC2530 is a wireless sensor network produced by Texas Instruments that supports the IEEE802.15.4 standard protocol. It is a true system-on-a-chip (SoC) solution. The ZigBee network established by this solution has the advantages of low cost, high efficiency and good performance [12], [13].

The ZigBee module includes a power supply circuit, an antenna module circuit, a sensor circuit module, and an LED circuit module. The main functions of each part are: the LED circuit is mainly used to judge whether the ZigBee networking is successful; the antenna circuit is mainly responsible for transmitting the data collected by the sensors; the power supply circuit is responsible for supplying power to the ZigBee module; and the USB to serial port circuit is responsible for implementing the communication function of the serial port debugging assistant of the ZigBee module and the computer terminal. The hardware structure of the ZigBee module is shown in Fig. 3

\section{B. Hardware design of sensor module}

The temperature and humidity sensor node uses a DHT11 temperature and humidity sensor that has high sensitivity, low cost and a small number of pins. The ultrasonic sensor node uses an HC-SR04 ultrasonic ranging module capable of making non-contact ranging in the $0-4.5 \mathrm{~m}$ range. The onboard temperature sensor is used to correct the ranging result to make the ranging result more accurate. In the meantime, the sensor module has multiple communication modes including GPIO and serial port that operate in a stable and reliable manner. The human body infrared sensor node uses an HC-SR

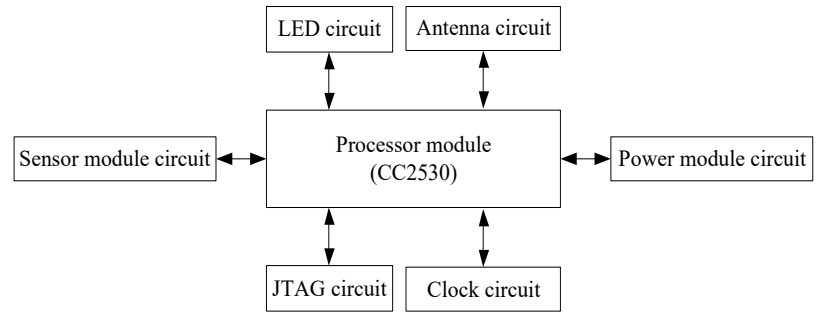

Fig. 3. Hardware structure of ZigBee module.

501 human body infrared sensor module that is sensitive, reliable, and consumes very little energy. These sensors protect the cultural relics by detecting the range of motion of the human body. The smoke sensor node uses a MQ-5 probe that is highly sensitive to liquefied gas, natural gas and city gas, and has long-term service life and stability. It can be used to monitor the gas concentration of the environment in which the cultural relics are placed in order to issue early warning and to prevent damage to cultural relics.

\section{Hardware design of system ARM gateway}

The STM32 processor chip used in this article has a model number of STM32F103VET6. This chip includes the core device of STM32. The Cortex-M3 core transmits data through the various buses, peripherals, and memories, and the bus matrix accomplishes the switching operation function. This structure makes it easier for the core to handle complex data [14].

The gateway of the cultural relics protection system chooses STM32 as the main control center. The gateway must not only be connected to the ZigBee coordinator through the serial port, but also be connected to the WiFi module and the GSM module through the serial port circuit. This requires the presence of at least three serial port circuits for the gateway portion of the system. STM32F103VET6 supports five serial ports, which are respectively UART0, UART1, UART2, UART3, and UART4. In the hardware design of ARM gateway, serial circuit 1 is connected with ZigBee coordinator, serial circuit 2 is connected with $\mathrm{WiFi}$ module, and serial circuit 3 is connected with GSM module, thus realizing the communication between ZigBee network and host computer. The block diagram of the ARM gateway is shown in Fig. 4.

The YYROBOT_SIM800 module is a high performance and effective industrial grade GSM/GPRS module (development board) [15]. The GSM module and the ARM gateway are connected through the serial port circuit 2 . When the ARM gateway detects a change of the distance, the GSM module will send out a warning message to the relevant user (the content of the short message is "The cultural relic is not safe!").

\section{WiFi module selection}

Since the system transmits only a small amount of data, an ESP8266 WiFi module is selected. The ESP8266 module has a high-performance wireless SOC, which is good news for mobile platform designers. It offers maximum utility at the lowest cost, making it possible to embed WiFi functionality into other systems. 


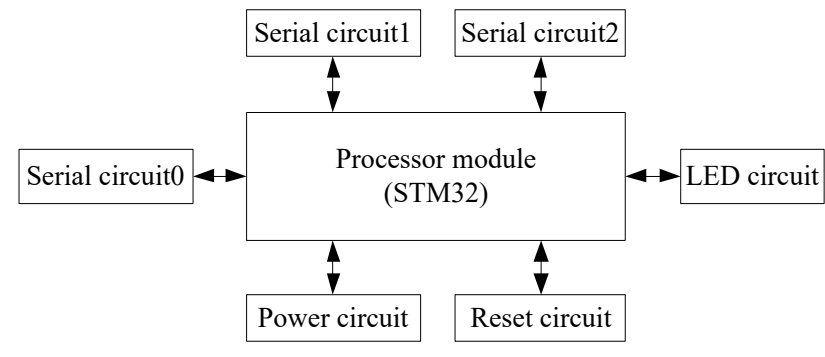

Fig. 4. Block diagram of the ARM gateway structure.

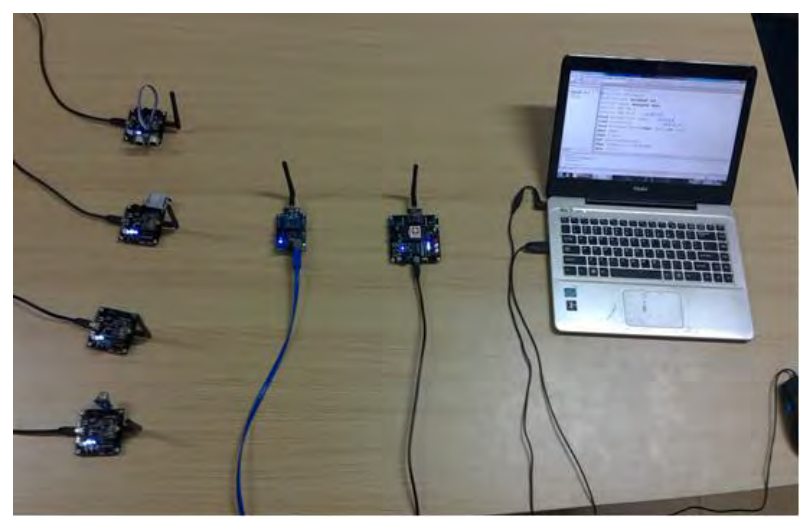

Fig. 5. Implementation of the system.

\section{E. Physical demonstration of system}

The programmed code is first burned into the coordinator and the terminal device, and then the program for the STM32 gateway and the interface with the host computer are developed. The system is then set up to run. The actual operation of the system is shown in Fig. 5.

\section{SOFTWARE DESIGN}

\section{A. Software design of ZigBee wireless communication network}

In this article, the software development of the ZigBee module was done in an IAR Embedded Workbench development environment and through a combination with the ZigBee protocol stack. The ZigBee protocol stack Z-Stack complies with the ZigBee 2006 specification and supports platforms based on the CC2530 transceiver and the TI MSP430 ultralow-power microcontroller. The Z-Stack includes a fullfeatured protocol stack for network topology, which plays an important role in the highly competitive industrial arena [16 17].

The task of the ZigBee terminal device is to collect data from the various sensors and to execute the control commands sent by the coordinator. The data information collected from the sensors is then sent to the coordinator through the wireless sensor module.

\section{B. Software design of ARM gateway}

The ARM gateway software of the cultural relics protection system is developed using MDK Keil, which is an integrated development environment for STM32 applications. The uploading of the data from the ESP8266 WiFi module to the cloud server relies mainly on the HTTP protocol. The same HTTP protocol is also required when the host computer accesses the cloud server to obtain data. This paper mainly uses the GET and POST request commands. The GET command is used to obtain resources, while the POST request command is used to execute operations. The parameters are provided to the server through URL check of the querystring and the body of the message. The ARM gateway is the communication hub of the entire cultural relics protection system, it plays an important role in the connection between the ZigBee communication network and the host computer. Firstly, the STM32 configures the WiFi module to establish a connection with the host computer after initializing each port in the ARM gateway programming. If the command sent by the host computer is received, the serial port 2 interrupt service routine is executed. Next, the command is sent to the ZigBee coordinator through the serial port 1. After the ZigBee communication network part performs corresponding processing, the ZigBee coordinator sends the data collected by the sensor to the STM32 via the serial port 2. Finally, the STM32 transmits the data to the server via the WiFi module. In addition, if the STM32 controller determines that the distance collected by the ultrasonic ranging sensor is significantly larger or smaller than the set threshold, the serial port 3 sends an alarm message to the corresponding user through the GSM module. The workflow of the ARM gateway STM32 is shown in Fig. 6.

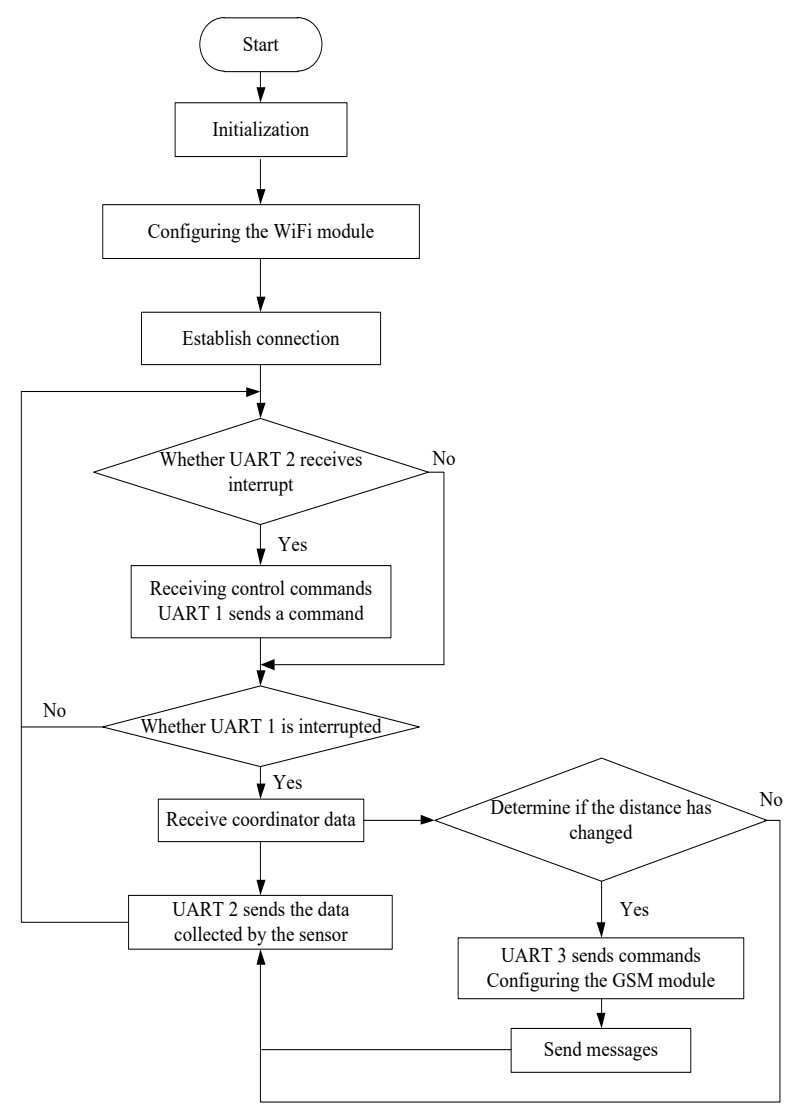

Fig. 6. Flow chart of ARM gateway STM32. 
In order to have data transmission capability in a wide area network (WAN), the cultural relics protection system of this paper uses the cloud server platform "lewei50" as the cloud server for the Internet of Things. Four sensors need to be added to the server so that data may be transmitted to the cloud server using the HTTP protocol through STM32 and the WiFi module. Host computer software design

The development environment of the host computer of the cultural relics protection system is the Visual Studio 2012 (referred to as VS2012), and the computer program is written in $\mathrm{C \#}$ language in order to achieve the capabilities of accessing cloud server, data transmission and local storage. The computer interface of the cultural relics system mainly uses Windows forms application design to realize the interaction between human and computer in a graphical way. The system is developed using $\mathrm{C \#}$ language, the language developed by the development teamsled by Microsoft's Anders Hejlsberg and Scott Wiltamuth specifically for the .NET platform. The host computer application mainly includes the following parts: the login interface and the main interface. The main interface includes temperature and humidity monitoring, security monitoring, gas monitoring and distance monitoring. The running status of the host computer is as follows: running login window, entering user name and password, entering the main interface window and opening the service; establishing the connection, accessing the cloud server with HTTP protocol, so as to obtain the data of each sensor; displaying in the main window interface and realizing local storage. The block diagram of the host computer modules is shown in Fig. 7.

\section{CONCLUSIONS}

In this paper, a practical ZigBee and WiFi-based cultural relics protection system is designed. The monitoring of cultural relics is achieved through the four sensors carried by the terminals of the ZigBee wireless communication network. Cloud transmission of data is achieved by connecting the ZigBee coordinator and the ARM gateway, and a determination is made on whether the cultural relic has moved. If there is a movement, the mobile GSM module will send a text message to inform the user. Finally, computer programs are written for the host computer to access the cloud server and to remotely monitor the cultural relics. This design has achieved the expected capability and the system has broad prospects for future applications.

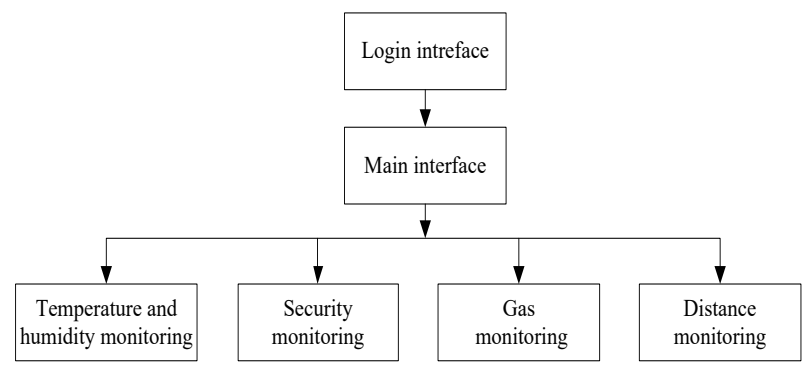

Fig. 7. Block diagram of host computer function modules.

\section{ACKNOWLEDGMENT}

The authors are grateful to the Science Foundation of China for its support of this research. This work was supported by the National Natural Science Foundation of China under Grant No.61461036 and No.61761033.

\section{REFERENCES}

[1] S. Farahani, ZigBee wireless networks and transceivers. Newnes, 2011.

[2] D. Culler, D., D. Estrin, and M. Srivastava, "Guest editors' introduction: Overview of sensor networks," Computer, vol. 37, issue 8, pp. 41-49, 2004.

[3] C. Buratti, A. Conti, D. Dardari, et al., "An overview on wireless sensor networks technology and evolution," Sensors, vol. 9, issue 9, pp. 68696896, 2009

[4] S. Tilak, N. B. Abu-Ghazaleh, and W. Heinzelman, "Infrastructure tradeoffs for sensor networks," Proceedings of the 1st ACM international workshop on Wireless sensor networks and applications. ACM, 2002.

[5] K. C. Huang, Y. S. Yen, et al., "for Wireless Sensor Networks," Journal of software, vol. 4, issue 7, pp. 1282-1291, 2003.

[6] A. Mainwaring, D. Culler, J. Polastre, et al., "Wireless sensor networks for habitat monitoring," Proceedings of the 1st ACM international workshop on Wireless sensor networks and applications. Acm, pp. 88-97, 2002.

[7] J. Polastre, R. Szewczyk, A. Mainwaring, et al., "Analysis of wireless sensor networks for habitat monitoring," Wireless sensor networks. Springer, Boston, MA, pp. 399-423, 2004.

[8] G. J. Pottie, and W. J. Kaiser, "Wireless integrated network sensors," Communications of the ACM, vol. 43, issue 5, pp. 51-58, 2000.

[9] M. Terada, "Application of ZigBee sensor network to data acquisition and monitoring," Measurement Science Review, vol. 9, issue 6, pp. 183186, 2009.

[10] J. F. Su, S. H. Nie, and S. C. Qu, "Design of Multi-Point Wireless Pulse Measuring System Base on CC2530/Zigbee Network," Applied Mechanics and Materials, vol. 401, pp. 1200-1203, 2013.

[11] Y. M. Wang, "The Internet of Things smart home system design based on ZigBee/GPRS technology," Applied Mechanics and Materials, vol. 263, pp. 2849-2852, 2013.

[12] S. Guo, C. Wang, Q. Si, et al., "GW27-e1174 A remote monitoring system of electrocardiogram based on GPRS network," Journal of the American College of Cardiology, vol. 68(Suppl.), pp. 187-188, 2016.

[13] B. Xu,Y. J. Zheng, Y. X. Yin, et al., "Temperature and Humidity Monitoring System Based on CC2530," Applied Mechanics and Materials, vol. 333, pp. 460-464, 2013.

[14] L. Lin, X. Li, and W. Gu, "PM2. 5 monitoring system based on ZigBee wireless sensor network," IOP Conference Series: Earth and Environmental Science, vol. 69, no. 1, pp. 012094, 2017.

[15] Y. Huang, L. Hu, D. Yang, et al., "Air-Sense: indoor environment monitoring evaluation system based on ZigBee network," IOP Conference Series: Earth and Environmental Science, vol. 81, no. 1, pp. $012208,2017$.

[16] L. Xiaohui, "Design and Implementation of Wireless Voltage Monitoring System Based on Zigbee," International Journal of Information Technology and Web Engineering (IJITWE), vol. 124, issue 3, pp. 83-96, 2017.

[17] T. A. Alhmiedat, F. Omar, A. A. Taleb, et al., "Road safety and energy saving proposed system: A ZigBee WSN approach," International Journal of Online Engineering (iJOE), vol. 11, issue 2, pp. 55-59, 2015. 